University of Nebraska - Lincoln

DigitalCommons@University of Nebraska - Lincoln

4-2009

\title{
Recent Progress in Acoustic Travel-Time Tomography of the Atmospheric Surface Layer
}

Vladimir E. Ostashev

Department of Physics, New Mexico State University, Las Cruces, NM, USA

Sergey N. Vecherin

Department of Physics, New Mexico State University, Las Cruces, NM, USA

D. Keith Wilson

U.S. Army Engineer Research and Development Center, Hanover, NH, USA

Astrid Ziemann

University of Leipzig, Institute for Meteorology, Leipzig, Germany

George H. Goedecke

Department of Physics, New Mexico State University, Las Cruces, NM, USA

Follow this and additional works at: https://digitalcommons.unl.edu/usarmyresearch

Part of the Operations Research, Systems Engineering and Industrial Engineering Commons

Ostashev, Vladimir E.; Vecherin, Sergey N.; Wilson, D. Keith; Ziemann, Astrid; and Goedecke, George H., "Recent Progress in Acoustic Travel-Time Tomography of the Atmospheric Surface Layer" (2009). US Army Research. 45.

https://digitalcommons.unl.edu/usarmyresearch/45

This Article is brought to you for free and open access by the U.S. Department of Defense at DigitalCommons@University of Nebraska - Lincoln. It has been accepted for inclusion in US Army Research by an authorized administrator of DigitalCommons@University of Nebraska - Lincoln. 


\title{
Recent progress in acoustic travel-time tomography of the atmospheric surface layer
}

\author{
Vladimir E. Ostashev ${ }^{1,2 *}$, Sergey N. Vecherin ${ }^{1,3}$, D. Keith Wilson ${ }^{3}$, Astrid Ziemann $^{4}$ \\ and GEORGE H. GOEDECKE ${ }^{1}$
}

${ }^{1}$ Department of Physics, New Mexico State University, Las Cruces, NM, USA

${ }^{2}$ NOAA/Earth System Research Laboratory, Boulder, CO, USA

${ }^{3}$ U.S. Army Engineer Research and Development Center, Hanover, NH, USA

${ }^{4}$ University of Leipzig, Institute for Meteorology, Leipzig, Germany

(Manuscript received October 6, 2008; in revised form January 19, 2008; accepted January 19, 2008)

\begin{abstract}
Acoustic tomography of the atmospheric surface layer (ASL) is based on measurements of the travel times of sound propagation between sources and receivers which constitute a tomography array. Then, the temperature and wind velocity fields inside the tomographic volume or area are reconstructed using different inverse algorithms. Improved knowledge of these fields is important in many practical applications. Tomography has certain advantages in comparison with currently used instrumentation for measurement of the temperature and wind velocity. In this paper, a short historical overview of acoustic tomography of the atmosphere is presented. The main emphasis is on recent progress in acoustic tomography of the ASL. The tomography arrays that have been used so far are discussed. Inverse algorithms for reconstruction of the temperature and wind velocity fields from the travel times are reviewed. Some results in numerical simulations of acoustic tomography of the ASL and reconstruction of the turbulence fields in tomography experiments are presented and discussed.

\section{Zusammenfassung}

Die akustische Tomographie der atmosphärischen Bodenschicht basiert auf Messungen der Laufzeit von Schallwellen zwischen Sendern und Empfängern, welche ein tomographisches Messfeld bilden. Anschließend werden dann die Temperatur- und Windgeschwindigkeitsfelder innerhalb eines tomographischen Volumens oder einer Fläche rekonstruiert, wobei verschiedene inverse Algorithmen angewendet werden können. Eine verbesserte Kenntnis dieser meteorologischen Felder ist für viele praktische Anwendungen bedeutsam. Tomographische Verfahren haben bestimmte Vorteile gegenüber den momentan genutzten Messverfahren für die Temperatur und Windgeschwindigkeit. In dieser Veröffentlichung wird ein kurzer Überblick zur Entwicklung der akustischen Tomographie der Atmosphäre präsentiert. Der Schwerpunkt der Arbeit liegt auf der Darstellung des aktuellen Fortschritts in der akustischen Tomographie der atmosphärischen Bodenschicht. Die bisher genutzten tomographischen Messfelder werden vorgestellt. Inverse Algorithmen für die Rekonstruktion von Temperatur- und Windgeschwindigkeitsfeldern aus akustischen Laufzeiten werden bewertet. Einige Resultate der numerischen Simulation der akustischen Tomographie der Bodenschicht und der Rekonstruktion von turbulenten Feldern meteorologischer Größen in tomographischen Experimenten werden dargestellt und diskutiert.
\end{abstract}

\section{Introduction}

The idea of acoustic tomography of the atmosphere is similar to that in medicine where ultrasound waves or $\mathrm{X}$-rays probe a particular organ of a human body resulting in an "image" of that organ. In the case of acoustic tomography of the atmospheric surface layer (ASL), sound waves are used for measuring the travel times $\tau_{n}$ of sound propagation between different pairs of sources (speakers) and receivers (microphones). Here, the subscript $n=1,2, \ldots N$ indicates a particular sound propagation path, where $N$ is the number of such paths. Then, the temperature $T$ and wind velocity $\mathbf{v}$ fields within the tomographic volume or area are reconstructed with different inverse algorithms.

\footnotetext{
${ }^{*}$ Corresponding author: Vladimir E. Ostashev, NOAA/EarthSystem Research Laboratory Physical Sciences Division, R/PSD 3325 Broadway, Boulder, CO 80305,USA, e-mail: Vladimir.Ostashev@ noaa.gov
}

Improved knowledge about the $T$ and $\mathbf{v}$ fields is important in many applications such as boundary layer meteorology, theories of turbulence, visualization of different dynamic processes in the ASL, experimental verification of large eddy simulation (LES), studies of electromagnetic and sound wave propagation through a turbulent atmosphere, etc. Currently, the temperature and wind velocity in the ASL are measured by conventional meteorological devices (e.g. ultrasonic anemometers/thermometers) or by volume imaging lidars. Acoustic tomography has certain advantages in comparison with both techniques. For example, conventional meteorological devices can have distinct inertia, can be affected by radiation, and can disturb the temperature and wind velocity fields; acoustic tomography does not have these shortcomings. Furthermore, acoustic tomography requires fewer sensors per unit data than do conventional meteorological devices. 
The first experimental implementation of acoustic tomography of the ASL was done by WILSON and THOMSON (1994) at the beginning of the 1990's. An overview of initial efforts in acoustic travel-time tomography of the atmosphere is presented in WILSON et al. (2001). In this decade, many important and interesting results have been obtained in acoustic tomography of the ASL. The main goal of the present paper is to present a brief overview of the recent progress in this field.

Note that acoustic travel-time tomography has also been used for remote sensing of the temperature and wind velocity fields above the ASL, though the term tomography was not always used. From the beginning of the 20th century until World War II, a large effort was undertaken by scientists from several European countries to use sound and infrasound waves generated by powerful explosions on the ground for remote sensing (tomography) of the vertical profiles of the temperature $T$ and wind velocity $\mathbf{v}$ in the atmosphere up to the heights of about $100 \mathrm{~km}$, e.g. see Cox (1949), DUKKERT (1931), OSTASHEV (1997) and references therein. Such remote sensing was important since no instrumentation was available at that time for measurements of the $T$ and $\mathbf{v}$ profiles in the upper atmosphere. Sound waves due to powerful explosions on the ground are refracted in the atmosphere by the temperature and wind velocity, can have turning points at some heights and come back to the ground. Measuring the travel times, velocities, and angles of arrival of these waves on the ground and assuming that the atmosphere is stratified, scientists tried to reconstruct the $T$ and $\mathbf{v}$ profiles in the upper atmosphere. Since the 1940-1950s, this effort in acoustic tomography of the upper atmosphere has been mostly abandoned due to a wide use of rockets and other techniques for measurements of parameters of the upper atmosphere. Still some research in this field has been continued (CRARY, 1950; KULICHKOV and BUSH, 2001). Furthermore, considerable effort was devoted to studies of infrasound propagation in the upper atmosphere due to nuclear explosions (PIERCE et al., 1971).

Currently, there is an opportunity to resume acoustic tomography of the upper atmosphere. The opportunity comes with a global network consisting of a large number of infrasound and seismic stations (already existing and many to be deployed) for verifying compliance with the Comprehensive Nuclear-Test-Ban Treaty (www.ctbto.org). Note that seismic stations can also detect infrasound waves (GIBBONS et al., 2007). These infrasound and seismic stations routinely measure infrasound signals from meteors, severe storms, mining explosions, and other human-caused or natural phenomena (BEDARD and GEORGES, 2000; MCKENNA et al., 2007). By determining the velocities, angles of arrival, and, in some cases, travel times of these waves, one can then try to solve an inverse problem: reconstruction of the $T$ and $\mathbf{v}$ fields in the atmosphere.

Finally note that acoustic tomography can be employed for remote sensing of the temperature and

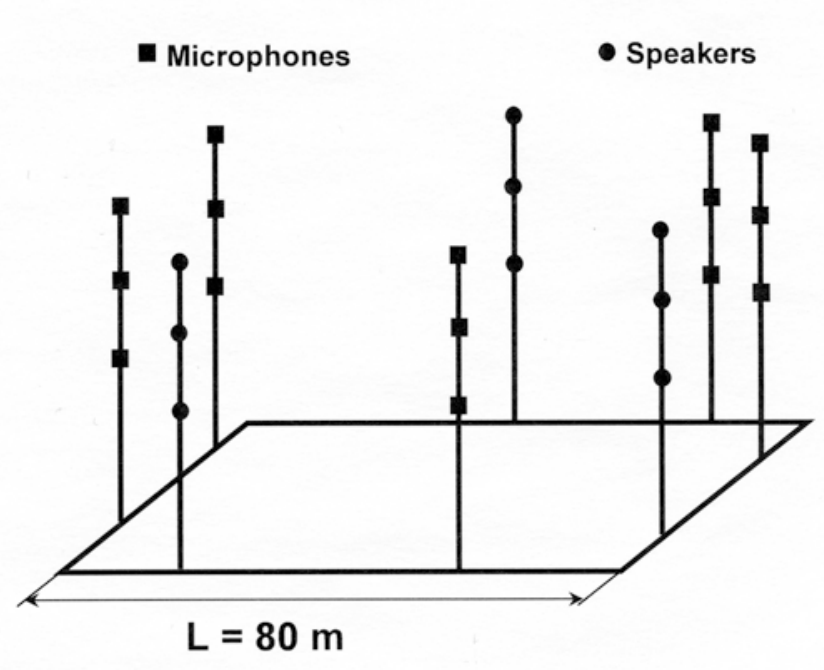

Figure 1: Schematic of the BAO acoustic tomography array.

wind velocity fields in the atmospheric boundary layer $(\mathrm{ABL})$ and in the troposphere. Most of the schemes for such acoustic tomography, experimentally implemented (ChunChuzov et al., 2007) or theoretically suggested (GREENFIELD et al., 1974; OSTASHEV, 1982), are similar to those for acoustic tomography of the upper atmosphere with both sound sources (propane cannons, small explosions, loudspeakers, etc.) and receivers located on the ground. A somewhat different scheme for acoustic tomography of the lower troposphere was suggested in OSTASHEV et al. (2000) and WILSON et al. (2001), in which noise from commercial airplanes is used as the sound source. This noise is recorded by a set of microphones located on the ground to determine the time delays of sound propagation between the airplane and different microphones. Then, the $T$ and $\mathbf{v}$ profiles can be reconstructed using an inverse algorithm.

The paper is organized as follows. In Sec. 2, the experimental arrays for acoustic tomography of the ASL that have been used so far are discussed. The forward and inverse problems in acoustic tomography of the ASL are considered in Sec. 3. In Sec. 4, the recently developed time-dependent stochastic inversion (TDSI) algorithm is discussed. In Sec. 5, the results presented in the paper are summarized.

\section{Acoustic tomography arrays}

In this section, we consider the experimental arrays that have been used for acoustic tomography of the ASL.

The first array for acoustic tomography of the ASL was built at the Pennsylvania State University at the beginning of the 1990s (WILSON and THOMSON, 1994). In the array, three speakers and five microphones were located a few meters above the ground along the perimeter of a square with a side length $200 \mathrm{~m}$. Frequency 
modulated sound waves were used as signals. By crosscorrelating the transmitted signals with those recorded by microphones, the travel times $\tau_{n}$ of sound propagation between different pairs of speakers and microphones were determined. The total number of travel times was $N=3 \times 5=15$. Then, the stochastic inversion (SI) algorithm was used to reconstruct the temperature $T$ and wind velocity $\mathbf{v}$ fields inside the tomographic area. The Penn State array was dismantled after a short period of operation.

In the mid 1990s, a portable array for acoustic tomography of the ASL was built at the Institute of Meteorology, the University of Leipzig, Germany. In this array, speakers and microphones were mounted on tripods 2 $\mathrm{m}$ above the ground. The size of the array varied and was of the order of several hundred meters. The number of speakers and microphones also varied and could be as large as 8 and 12, respectively. Speakers transmitted two short bursts of a harmonic signal separated by about $20 \mathrm{~ms}$; the frequency range of the transmitted signal was around $1 \mathrm{kHz}$. Since the mid 1990s, this tomography array has been used in about 10 experimental campaigns (ARNOLD et al., 1999, 2001, 2004; RAABE et al., 2003, 2005; TETZLAFF et al., 2002; WEINBRECHT et al., 2004; ZIEMANN et al., 1999, 2001, 2002).

An array for acoustic tomography of the ASL has recently been built at the Boulder Atmospheric Observatory (BAO) which is a premier meteorological site (KAIMAL and CAYNOR, 1983) with many instruments for measuring parameters of the ABL. Construction of the BAO acoustic tomography array has been a collaborative effort by New Mexico State University, the Earth System Research Laboratory of NOAA, and the Cooperative Institute for Research in Environmental Sciences of the University of Colorado at Boulder. A schematic of the array is shown in Fig. 1. The array consists of three speaker and five microphone towers located along the perimeter of a square with the side length $L=80$ $\mathrm{m}$. Speakers and microphones are located on the towers at three levels ranging from about 3 to $9 \mathrm{~m}$. Speakers and microphones are connected via underground cables with a central command and data acquisition computer, which is located inside a small modular building.

The BAO tomography array allows one to measure the travel times $\tau_{n}$ of sound propagation between different pairs of speakers and microphones shown in Fig. 1. Until now, only the speakers and microphones at the upper level of the array have been used in transmission and reception of acoustic signals, thus enabling 2D horizontal slice tomography. In what follows, the BAO array operating with transducers only at the upper level is called the 2D BAO acoustic tomography array. A schematic of the 2D BAO array and sound propagation paths between different pairs of speakers and microphones are shown in Fig. 2(a). Note that speakers and microphones are permanently installed on the towers of the BAO array. This feature of the array allows continual monitoring of the $T$ and $\mathbf{v}$ fields inside the tomographic volume or area. All previous arrays for acoustic tomography were dis- mantled after a few days of operation. Several acoustic tomography experiments have already been carried out with the BAO array. Some of the results obtained are reported in OSTASHEV et al. (2008).

In tomography, the quality of reconstruction of unknown fields improves with increasing number of sources and receivers. To increase the number of speakers and microphones in the BAO tomography array, several additional transducer towers could be placed along the perimeter of the array. However, this is relatively expensive. Furthermore, a large number of towers can distort the $T$ and $\mathbf{v}$ fields inside the tomography array. Therefore, another way to increase the number of transducers in the 2D BAO array was suggested. Both a speaker and a microphone will be installed at the upper level of all towers of the array, as schematically shown in Fig. 2(b). The 2D BAO array shown in Fig. 2(b) will enable us to do reciprocal sound transmission (sound transmission in opposite directions) similar to that used in ultrasonic anemometers/thermometers. The number $N$ of different sound propagation paths (and, hence, the travel times $\tau_{n}$ ) which are achieved with this array is 56 , significantly greater than 15 propagation paths (travel times $\tau_{n}$ ) of the current 2D BAO array shown in Fig. 2(a).

A somewhat different scheme of acoustic tomography of the ASL was proposed by SPIESBERGER and FRISTRUP (1990) and experimentally implemented by SPIESBERGER (1999). In this scheme, an array of microphones located near the ground recorded calling birds. The positions of birds and time of acoustic calls were not known. By measuring the time differences between an acoustic call arrival at different microphones and then using an inverse algorithm developed, it was possible to locate a calling bird and to reconstruct the $T$ and $\mathbf{v}$ fields inside the volume between the bird and the microphones.

Finally note that scientists from the University of Leipzig have also built a scalable, indoor prototype of their outdoor array for acoustic tomography (BARTH et al., 2004, 2007; HolsTEIN et al., 2004). The horizontal size of the indoor array varied from $1 \mathrm{~m}$ to $20 \mathrm{~m}$. The transmitted signal was a pseudo-random noise. The indoor array was built to test acoustic tomography in controlled environments and to compare different algorithms for reconstruction of the $T$ and $\mathbf{v}$ fields. It can also be used for studies of turbulence in wind channels and over heated surfaces (BARTH et al., 2007).

\section{Forward and inverse problems in acoustic tomography of the ASL}

\subsection{Forward problem}

The forward problem in acoustic tomography of the ASL is to express the travel time $\tau_{n}$ of sound propagation along a particular sound propagation path in terms of the temperature $T(\mathbf{r})$ and wind velocity $\mathbf{v}(\mathbf{r})$ fields. If it is not stated otherwise, in what follows we will consider $2 \mathrm{D}$, horizontal slice tomography similar to those 
(a)

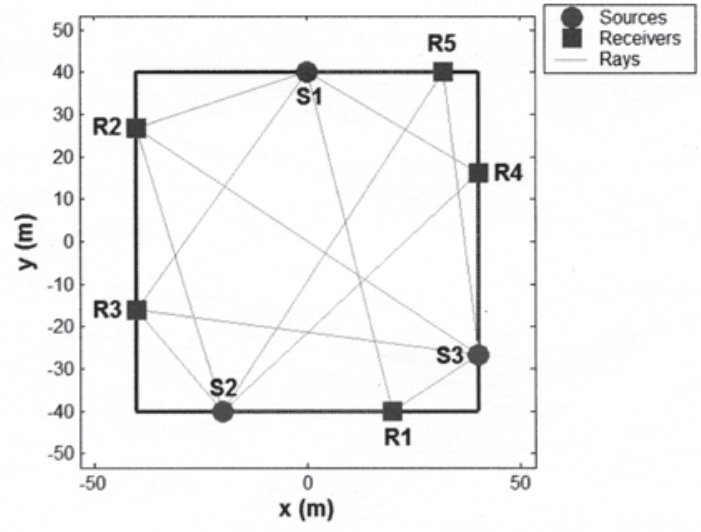

(b)

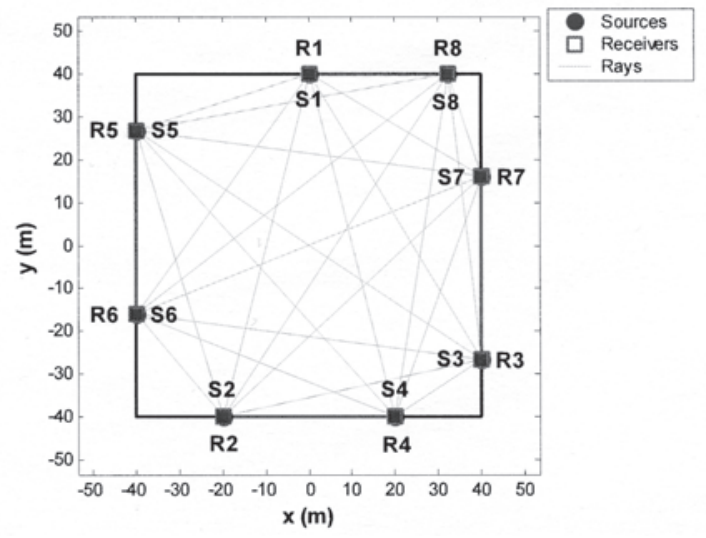

Figure 2: (a) Schematic of the 2D BAO acoustic tomography array. (b) Schematic of the $2 \mathrm{D}$ BAO acoustic tomography array with reciprocal sound transmission. In both figures, S1-S8 are speakers and R1-R8 are receivers (microphones). Solid lines indicate sound propagation paths.

schematically shown in Figs. 2(a) and (b). In this case, the vector $\mathbf{r}=(x, y)$ indicates the Cartesian coordinates in a horizontal plane. Using results from geometrical acoustics in moving inhomogeneous media (see Sec. 3.3.2 in OSTASHEV, 1997), the following expression for $\tau_{n}$ is obtained:

$$
\tau_{n}=\int_{L_{n}} \frac{d l}{u_{g r}}
$$

Here, the integration is over the $n$th sound propagation path, $L_{n}$ is the length of the path, and $u_{g r}=|c+\mathbf{v}|$ is the group velocity of the sound wave, where the adiabatic sound speed $c=\sqrt{\gamma R_{a} T}$. In the latter formula, $\gamma=1.4$ is the ratio of specific heats, $R_{a}$ is the gas constant for air, and $T$ is the acoustic virtual temperature, which is used in the literature devoted to ultrasonic anemometers/thermometers, RASS, and acoustic tomography. (The formula $c=\sqrt{\gamma R_{a} T}$ can be considered as a definition of the acoustic virtual temperature.) $T$ is related to the thermodynamic temperature $T_{t h}$ by (OSTASHEV, 1997): $T=(1+0.51 q) T_{t h}$, where $q$ is the specific humidity. Thus, acoustic tomography actually allows one to reconstruct the acoustic virtual tem- perature. For simplicity, through the text, "temperature" means "acoustic virtual temperature".

Formula (3.1) is a non-linear expression relating $\tau_{n}$ with $T$ and $\mathbf{v}$ that significantly complicates solution of the inverse problem. It was shown in WILSON and THOMSON (1994) that (3.1) can be linearized if the size of the tomographic area is less than a few hundred meters. Furthermore, it is worthwhile to represent the temperature and wind velocity fields as the following sums: $T(\mathbf{r})=T_{0}+\tilde{T}(\mathbf{r})$ and $\mathbf{v}(\mathbf{r})=\mathbf{v}_{0}+\tilde{\mathbf{v}}(\mathbf{r})$. Here, $T_{0}$ and $\mathbf{v}_{0}=\left(v_{0, x}, v_{0, y}\right)$ are the mean values (averaged over the tomographic area) of the temperature and wind velocity, $\tilde{T}(\mathbf{r})$ and $\tilde{\mathbf{v}}(\mathbf{r})=\left(\tilde{v}_{x}(\mathbf{r}), \tilde{v}_{y}(\mathbf{r})\right)$ are their fluctuating parts, and the subscripts $x$ and $y$ indicate the components of the wind velocity vector along the $x$ - and $y$-axes. As a result of such manipulations, formula (3.1) takes the form (VECHERIN et al., 2006a)

$$
\begin{gathered}
\tau_{n}=\frac{L_{n}}{c_{0}}\left(1-\frac{v_{0, x} \cos \phi_{n}+v_{0, y} \sin \phi_{n}}{c_{0}}\right)-\frac{1}{c_{0}} \int_{L_{n}} \\
{\left[\frac{\tilde{T}(\mathbf{r})}{2 T_{0}}+\frac{\tilde{v}_{x}(\mathbf{r}) \cos \phi_{n}}{c_{0}}+\frac{\tilde{v}_{y}(\mathbf{r}) \sin \phi_{n}}{c_{0}}\right] d l+\varepsilon_{n} .}
\end{gathered}
$$

Here, the integration is along the straight line connecting a source and receiver, $L_{n}$ is the length of this straight line, $\phi_{n}$ is the angle between the line and the $x$-axis, $c_{0}=\sqrt{\gamma R_{a} T_{0}}$ is the mean value of the adiabatic sound speed, and $\varepsilon_{n}$ corresponds to errors in measurements of travel times and transducer coordinates. Formula (3.2) is a desired solution of the forward problem of acoustic travel-time tomography of the ASL. It expresses the travel times $\tau_{n}$ in terms of the temperature $T(\mathbf{r})$ and wind velocity $\mathbf{v}(\mathbf{r})$ fields, and the length $L_{n}$ and angle $\phi_{n}$, the values of which can be determined if the transducer coordinates are known. Note that a 3D forward problem in acoustic tomography of the ASL is formulated similarly to equation (3.2), see VECHERIN et al. (2008a).

\subsection{Inverse problem}

The inverse problem in acoustic tomography of the ASL is to reconstruct the temperature and wind velocity fields inside a tomographic area (e.g., the tomographic area shown in Fig. 2) given the travel times $\tau_{n}$ and the values of $L_{n}$ and $\phi_{n}$. In other words, one has to solve equations (3.2) for $T(\mathbf{r})=T_{0}+\tilde{T}(\mathbf{r})$ and $\mathbf{v}(\mathbf{r})=\mathbf{v}_{0}+\tilde{\mathbf{v}}(\mathbf{r})$ given the values of $\tau_{n}, L_{n}$, and $\phi_{n}$.

Figure 3(a) depicts the magnitude and direction (indicated with arrows) of the wind velocity field $\mathbf{v}(\mathbf{r})=$ $\left(v_{x}(\mathbf{r}), v_{y}(\mathbf{r})\right)$ within the square $80 \times 80 \mathrm{~m}^{2}$ which coincides with the tomographic area of the $2 \mathrm{D}$ BAO array. The velocity field was obtained with a snapshot of LES for unstable conditions that had the spatial resolution of $4 \mathrm{~m}$ in the horizontal plane (for more details, see WILSON et al., 2007, and SULLIVAN et al., 1994). Thus, 
$20 \times 20=400$ values of the vector $\mathbf{v}=\left(v_{x}, v_{y}\right)$ (with linear interpolation between the grid points) were used to plot Fig. 3(a). The goal of the inverse problem in acoustic tomography of the ASL is to reconstruct the $T$ and $\mathbf{v}=\left(v_{x}, v_{y}\right)$ fields which are similar to that shown in this figure. If the tomographic area of the $2 \mathrm{D} \mathrm{BAO}$ array were divided into 400 cells (as in Fig. 3a), 1200 values of $T, v_{x}$, and $v_{y}$ should be reconstructed from only 15 travel times between 3 speakers and 5 microphones. This is a highly undetermined inverse problem. Note that, in acoustic tomography experiments that have been done so far and for those planned in the future, the number of sound propagation paths $N$ is less than about 100 . With this number of propagation paths, the inverse problem in acoustic tomography of the ASL remains highly undetermined if detailed reconstruction of the turbulence eddies is desired.

Several different approaches to a solution of the inverse problem in acoustic tomography of the ASL have been considered in the literature. One of these approaches is algebraic reconstruction, e.g. see ARNOLD et al. (2001), BARTH et al. (2004), JovANOVIC et al. (2006), RAABE et. al. (2005), VECHERIN et al. (2004), ZIEMANN et al. (2002). In this approach, the tomographic area is divided into $M$ cells (usually squares). Inside each cell, the values of the temperature $T_{m}$ and wind velocity $\mathbf{v}_{m}=\left(v_{x, m}, v_{y, m}\right)$ are assumed constant, where index $m=1,2, \ldots M$ indicates a particular cell. Then, the integrals in equations (3.2), where $n=1,2, \ldots N$, are evaluated analytically so that these equations become a set of algebraic equations for the unknown fields $T_{m}, v_{x, m}$, and $v_{y, m}$. The set can be solved for these fields using well- known methods, e.g. the least squares solution or the damped least squares solution. Such algebraic reconstruction allows one to accurately determine the unknown fields $T_{m}, v_{x, m}$, and $v_{y, m}$ if the inverse problem is overdetermined, i.e., if the number $N$ of data (travel times $\tau_{n}$ ) is larger than the number $3 M$ of the unknown fields $T_{m}, v_{x, m}$, and $v_{y, m}: N>3 M$. Since $N \lesssim 100$, in the acoustic tomography arrays that have been used until now, detailed reconstruction of the turbulence fields with the algebraic approach is infeasible. Similarly, it was found (COLLIER et al., 2004; VECHERIN et al., 2004) that detailed reconstruction of the turbulence fields is infeasible with the Monte-Carlo and basis function approaches.

In several papers (WILSON and THOMSON, 1994; WILSON et al., 2004; OSTASHEV et al. 2006a; VECHERIN et al., 2006a), the SI algorithm was used to reconstruct the turbulence fields in acoustic tomography of the ASL. This algorithm is particularly well suited for solutions of undetermined inverse problems, when $N<3 M$. The algorithm actually applies to the reconstruction of the fluctuating parts $\tilde{T}(\mathbf{r})$ and $\tilde{\mathbf{v}}(\mathbf{r})$ of the temperature and wind velocity fields. To reconstruct the mean values of these fields, $T_{0}$ and $\mathbf{v}_{0}$, in equations (3.2) the fluctuations are set to zero. The resulting set of equations is solved for $T_{0}$ and $\mathbf{v}_{0}$ using the least squares solu- tion. Numerical simulations of the BAO acoustic tomography array showed that the mean values of the temperature and wind velocity inside the tomographic volume or area are reconstructed with a good accuracy for both $2 \mathrm{D}$ and $3 \mathrm{D}$ geometries.

After the mean values $T_{0}$ and $\mathbf{v}_{0}$ are determined, equations (3.2) comprise an inverse problem for reconstruction of the fluctuations $\tilde{T}(\mathbf{r})$ and $\tilde{\mathbf{v}}(\mathbf{r})$. The main idea of the SI is to assume that $\tilde{T}(\mathbf{r})$ and $\tilde{\mathbf{v}}(\mathbf{r})$ are random fields with known spatial covariance functions of temperature and velocity fluctuations, $B_{T}(\mathbf{r})$ and $B_{i j}(\mathbf{r})$,respectively. Here, $i$ and $j$ are the indices corresponding to the two components of the wind velocity vector. Then, using formulas known in the literature (e.g. WILSON and THOMSON, 1994; VeCHERIN et al., 2006a), the fluctuations $\tilde{T}(\mathbf{r})$ and $\tilde{\mathbf{v}}(\mathbf{r})$ are calculated given the values of $\tau_{n}, L_{n}, \phi_{n}, T_{0}$, and $\mathbf{v}_{0}$. Moreover, the SI allows one to estimate expected errors in reconstruction of the fluctuations. The total temperature and velocity fields are found by adding fluctuations to the mean values.

Covariance functions $B_{T}(\mathbf{r})$ and $B_{i j}(\mathbf{r})$ depend on meteorological regimes of the ASL; their exact forms are not yet known. Therefore, in OSTASHEV et al. (2006a), different forms of the $B_{T}(\mathbf{r})$ and $B_{i j}(\mathbf{r})$ were used in numerical simulations of the 2D BAO tomography array: von Karman, Gaussian, exponential, and a new correlation function proportional to $\exp \left(-r^{2 / 3} / L^{2 / 3}\right)$, where $L$ is the outer length-scale of turbulence. The results obtained showed that the reconstructed temperature and wind velocity fields practically do not depend on the forms of the covariance functions if their outer length-scale $L$ is chosen properly. Thus, the SI algorithm itself is not sensitive to the forms of the covariance functions. It is worthwhile to use the Gaussian covariance functions of temperature and wind velocity fluctuations because then many calculations can be done analytically and numerical reconstruction of the $\tilde{T}(\mathbf{r})$ and $\tilde{\mathbf{v}}(\mathbf{r})$ fields is much faster. The Gaussian covariance function of temperature fluctuations is given by

$$
B_{T}^{G}(r)=\sigma_{T}^{2} \exp \left(-r^{2} / L^{2}\right),
$$

where $\sigma_{T}^{2}$ is the variance of temperature fluctuations. The Gaussian covariance functions of velocity fluctuations $B_{i j}^{G}(\mathbf{r})$ are given in OSTASHEV (1997). Numerical simulations of acoustic tomography of the ASL also showed that the smallest eddies that are reconstructed with SI are of the order of the outer length-scale $L$. However, a decrease in $L$ results in increasing errors in the reconstruction. (This result follows from the analysis of the diagonal elements of the error covariance matrix, see equation (22) in VECHERIN et al., 2006a, and from numerical simulations of acoustic tomography in WILSON et al., 2004). Therefore, in the SI algorithm, $L$ actually plays a role of an adjustable parameter and should be chosen as a trade-off between the size of the reconstructed eddies and the errors in the reconstruction. 

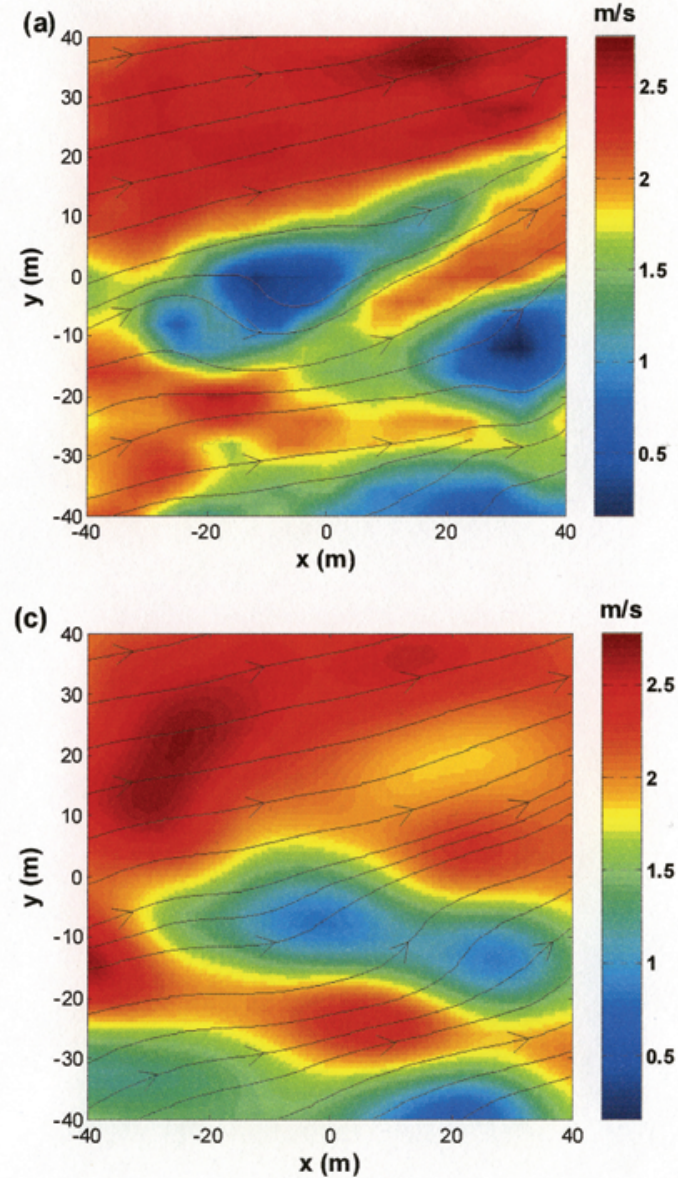
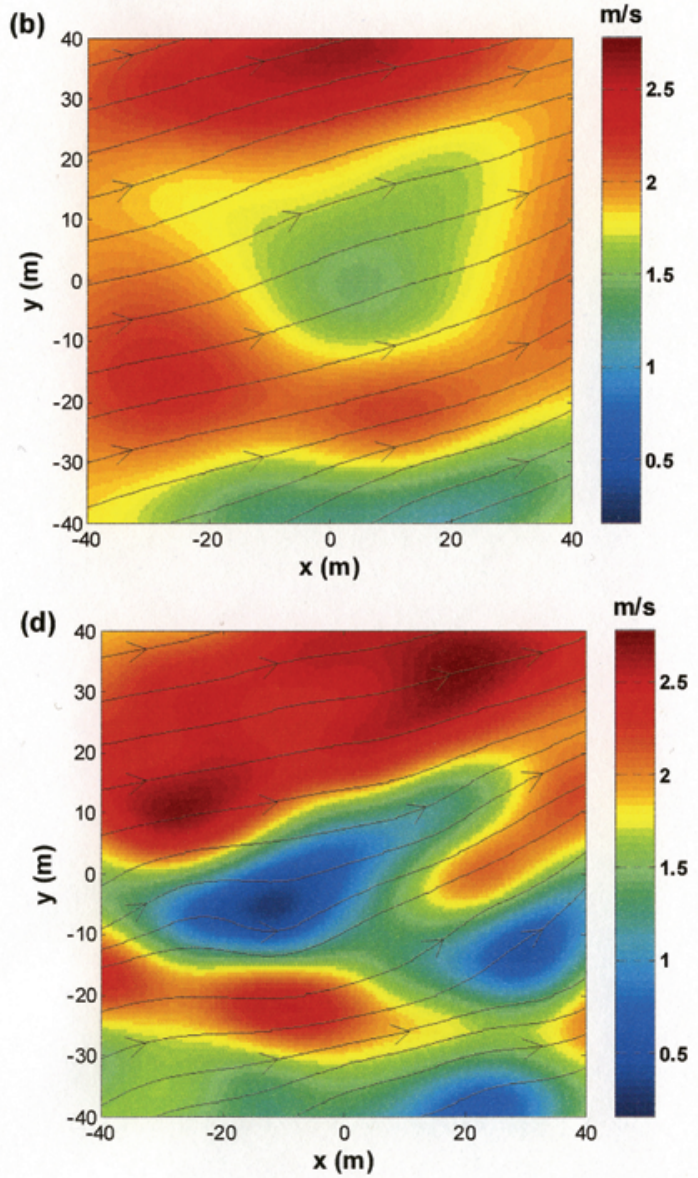

Figure 3: The wind velocity v field. Arrows indicate the direction of the velocity vector. (a) original LES field, (b) reconstructed with SI, (c) reconstructed with TDSI, (d) reconstructed with TDSI with reciprocal sound transmission.

At a first sight, SI has a disadvantage in comparison with the algebraic reconstruction, Monte-Carlo approach, and basis function approach, since it requires $a$ priori information about the covariance functions $B_{T}(\mathbf{r})$ and $B_{i j}(\mathbf{r})$. However, the numerical simulations showed that these covariance functions can be chosen as Gaussian covariance functions, in which $L$ plays the role of an adjustable parameter. Therefore, the only information that is needed in SI are the values of the variances of the temperature and wind velocity fluctuations, $\sigma_{T}^{2}$ and $\sigma_{v}^{2}$. These can be measured, for example, by placing an ultrasonic anemometer/thermometer inside a tomography array.

Figures 3(a) and (b) depict the wind velocity $\mathbf{v}$ field obtained with the LES and that reconstructed with SI in numerical simulations of the 2D BAO tomography array. In such numerical simulations, the forward problem is first solved. The travel times $\tau_{n}$ of sound propagation between different pairs of speakers and microphones shown in Fig. 2 (a) are calculated with equations (3.2) for the $\mathbf{v}$ field shown in Fig. 3(a) and the $T$ field obtained with the same LES. Then, these travel times $\tau_{n}$ are corrupted by noise $\varepsilon_{n}$ to mimic errors in measure- ments of the travel times and transducer coordinates; this completes a solution of the forward problem. In the inverse problem, the mean values of the temperature and velocity fields within the tomographic area are obtained with the least squares solution. Finally, the fluctuations $\tilde{T}(\mathbf{r})$ and $\tilde{\mathbf{v}}(\mathbf{r})$ are reconstructed with SI. A comparison between Figs. 3(a) and (b) reveals that SI is able to reproduce main spatial variations of the $\mathbf{v}$ field even though only 15 travel times were used in reconstruction. The root mean squared error (RMSE) in the reconstruction of the velocity field is $0.49 \mathrm{~m} / \mathrm{s}$, which is acceptable for some meteorological applications (ZIEMANN et al., 1999, 2002). Note that for the considered numerical example, an algebraic approach can reliably reconstruct the mean values of the $T$ and $\mathbf{v}$ fields inside four large squares with the size $40 \times 40 \mathrm{~m}^{2}$ so that a spatial structure of turbulence could not be revealed.

It should be noted that some velocity fields $\mathbf{v}(\mathbf{r})$ cannot be reconstructed if only the travel times $\tau_{n}$ of sound propagation are used in reconstruction. For reconstruction of such "invisible" fields, some other parameters of sound waves need to be measured. Examples of the invisible velocity fields can be found in JOHNSON et al. 
(1977), BRAUn and HAUCK (1991), and Jovanovic et al. (2006). However, the invisible fields presented in these papers are relatively simple and significantly differ from turbulent velocity fields in the atmosphere. Therefore, it would be interesting to study to what extent realistic velocity fields, e.g., the field shown in Fig. 3(a), can be reconstructed with the travel-time tomography.

\section{Time dependent stochastic inversion}

One of the reasons that SI yields a better reconstruction of the $T$ and $\mathbf{v}$ fields in acoustic tomography than other approaches is because SI takes into account the spatial correlation of these fields which does exist in the ASL. However, the temporal correlation of these fields, which also exists, is not employed in this algorithm. To account for both spatial and temporal correlations of the turbulent fields, the TDSI algorithm was recently developed (VECHERIN et al., 2006a).

The main idea of TDSI is to assume that the fluctuations $\tilde{T}(\mathbf{r}, t)$ and $\tilde{\mathbf{v}}(\mathbf{r}, t)$, where $t$ is time, are random fields with known spatial-temporal correlation functions $B_{T}(\mathbf{r}, t)$ and $B_{i j}(\mathbf{r}, t)$, respectively, and to measure the travel times $\tau_{n}$ repeatedly at the time moments $t_{1}, t_{2}$, ...t $t_{K}$. Here, $K$ is the number of such measurements. Then, using these $K$ sets of the travel times $\tau_{n}$ as input data, the $\tilde{T}(\mathbf{r}, t)$ and $\tilde{\mathbf{v}}(\mathbf{r}, t)$ fields are reconstructed with equations derived in VECHERIN et al. (2006a). Prior to reconstruction of the fluctuations, the mean fields $T_{0}\left(t_{k}\right)$ and $\mathbf{v}_{0}\left(t_{k}\right)$ at the time moments $t_{1}, t_{2}, \ldots t_{K}$ are determined with the least squares solutions similarly to that in the SI. The TDSI can also be used for reconstruction of 3D temperature and wind velocity fields (VECHERIN et al., 2008a). Note that the TDSI algorithm is somewhat similar to the vector Wiener filter which is used for the image reconstruction from several blurry images (OZKAN et al., 1992) and for medical tomography with two radionuclide (LINKS et al., 1996).

The spatial-temporal covariance functions $B_{T}(\mathbf{r}, t)$ and $B_{i j}(\mathbf{r}, t)$ that are needed in TDSI are calculated using either the hypothesis of perfectly frozen turbulence or the theory of locally frozen turbulence (TATARSKII, 1971). The latter theory generalizes the hypothesis of perfectly frozen turbulence to the case when turbulence is advected with a wind velocity which can change in space and time. In both cases, the spatial-temporal covariance functions $B_{T}(\mathbf{r}, t)$ and $B_{i j}(\mathbf{r}, t)$ are expressed in terms of the spatial covariance functions $B_{T}(\mathbf{r})$ and $B_{i j}(\mathbf{r})$, which are approximated by Gaussian covariance functions; see VECHERIN et al. (2006a) and VECHERIN et al. (2007).

The TDSI algorithm allows one to increase the amount of data (i.e., total number of the travel times $\tau_{n}$ ) without increasing the number of speakers and microphones. Numerical simulations of the BAO acoustic tomography array showed that TDSI results in significant increase in quality of reconstruction of the temperature $T$ and wind velocity $\mathbf{v}$ fields both for $2 \mathrm{D}$ and $3 \mathrm{D}$ geometries (OSTASHEV et al., 2006b; VECHERIN, 2007;
VECHERIN et al., 2008a). For example, Figs. 3(a) and (c) show the $\mathbf{v}$ field obtained with LES and that reconstructed with the TDSI. For this numerical example, five sets of the travel times $\tau_{n}$ were calculated with equation (3.2) when the LES field was moving through the tomographic area of the 2D BAO array shown in Fig. 2 (a). A comparison between Figs. 3(a), (b), and (c) reveals that the TDSI allows much better reconstruction of the $\mathbf{v}$ field than does SI. Most of the "fast" and "slow" velocity eddies which are seen in Fig. 3(a) are reconstructed in Fig. 3(c). The RMSE of this reconstruction is $0.37 \mathrm{~m} / \mathrm{s}$ which is less than that in SI.

Note that numerical simulations of the $2 \mathrm{D}$ and $3 \mathrm{D}$ BAO tomography arrays were also done for many other LES fields corresponding to stable, neutral, and unstable stratifications. Furthermore, in these simulations, the temperature and wind velocity fields were modeled with quasi-wavelets (WILSON et al., 2008). For all these realistic turbulence fields, it was shown that the TDSI yields better reconstruction than the SI.

The developed TDSI was applied (OSTASHEV et al., 2006b; VECHERIN et al., 2006b; VECHERIN et al., 2007; VECHERIN et al., 2008b) to the reconstruction of the temperature and wind velocity fields in some of the outdoor and indoor acoustic tomography experiments carried out by scientists from the University of Leipzig. The results obtained showed turbulent eddies of different sizes, which were reliably reconstructed, and their temporal evolution.

The TDSI algorithm is also used in reconstruction of the $T$ and $\mathbf{v}$ fields in acoustic tomography experiments with the 2D BAO array (OSTASHEV et al., 2008). To take full advantage of this inverse algorithm, the travel times are measured every $1.5 \mathrm{~s}$. Initial results in reconstruction of the temperature and wind velocity fields are presented in OSTASHEV et al. (2008).

Although the quality of reconstruction of the $T$ and $\mathbf{v}$ fields with the 2D BAO array is satisfactory, it can be further improved to decrease errors in the reconstruction and to capture smaller turbulent eddies than those shown in Fig. 3(c). Both these goals are important for applications. These goals can be achieved with the proposed 2D BAO tomography array with reciprocal sound transmission, depicted in Fig. 2(b). The number of different travel times that can be measured with this array is 56 which is significantly greater than 15 travel times for the current 2D BAO array plotted in Fig. 2(a). Numerical simulations showed (VECHERIN et al., 2008b) that such reciprocal transmission combined with the TDSI algorithm results in further improvement in reconstruction of the temperature and wind velocity fields and is worthwhile to pursue. For example, Fig. 3(d) depicts the $\mathbf{v}$ field reconstructed with the TDSI in numerical simulations of the 2D BAO array with reciprocal transmission. Comparison between Figs. 3(a), (c), and (d) clearly shows that the contours of the "fast" and "slow" velocity eddies and their amplitudes are reconstructed better if reciprocal transmission is employed. The RMSE in the reconstruction of the $\mathbf{v}$ field in Fig. 3(d) is only $0.21 \mathrm{~m} / \mathrm{s}$. 


\section{Conclusions}

In this paper, a brief historical overview of acoustic tomography of the atmosphere was first presented. The arrays for acoustic tomography of the ASL that have been used so far were discussed, including the recently built BAO tomography array. The forward and inverse problems in acoustic tomography of the ASL were formulated. Several approaches to solution of an inverse problem were considered: the algebraic reconstruction, Monte-Carlo approach, basis function approach, SI, and recently developed TDSI. Numerical simulations of acoustic tomography showed that, among these approaches, TDSI yields the best reconstruction of the temperature and wind velocity fields in acoustic tomography of the ASL. The TDSI algorithm has been used in reconstruction of the temperature and velocity fields in tomography experiments.

Acoustic tomography of the ASL has already been established as a unique technique for remote sensing of the temperature and wind velocity fields. In the future, it can have many interesting and important applications. One such application is continual monitoring of temporal changes in 2D and 3D temperature and wind velocity fields in the ASL. Such monitoring could allow us to gain new knowledge about the spatial structure of the $T$ and $\mathbf{v}$ fields and their temporal evolution in different meteorological regimes.

\section{Acknowledgments}

This material is partly based upon work that was supported by the U.S. Army Research Office under contracts W911NF-06-1-007 and DAAD19-03-1-0341.

\section{References}

Arnold, K., A. Ziemann, A. RaABe, 1999: Acoustic tomography inside the atmospheric boundary layer. - Phys. Chem. Earth (B) 24, 133-137.

- , 一, -, 2001: Tomographic monitoring of wind and temperature at different heights above the ground. - Acust. Acta Acust. 87, 703-708.

Arnold, K., A. Ziemann, A. RaAbe, G. Spindler, 2004: Acoustic tomography and conventional meteorological measurements over heterogeneous surfaces. - Meteor. Atmos. Phys. 85, 175-186.

Barth, M., A. RaAbe, P. Holstein, R. Muller, A. Ziemann, K. Arnold, D. Mackenzie, E. Starke, M. SEliger, 2004: Acoustic travel time tomography as a tool to investigate temperature distributions on different spatial scales. - Proc. 12th Intern. Symp. on Acoustic Remote Sensing (Cambridge, UK).

Barth, M., A. RaAbe, K. Arnold, C. Resagk, R. DU PUITS, 2007: Flow field detection using acoustic travel time tomography. - Meteorol. Z. 16, 443-450.

BedARD, A.J., T.M. GEORGES, 2000: Atmospheric infrasound. - Phys. Today 53, 32-37.
BRAUN, H., A. HAUCK, 1991: Tomographic reconstruction of vector fields. - IEEE Transactions on Signal Processing 39, 464-471.

Chunchuzov, I.P., S.N. KulichKov, V. Perepelkin, A. Ziemann, K. Arnold, A. KniffKa, 2007: Acoustic tomographic study of the mesoscale coherent structures in the lower atmosphere. - Proc. Meetings Acoust. http://scitation.aip.org/confst/ASA/pub/8/4pPA5.pdf

Collier, S.L., D.A. Ligon, J.M. Noble, E. PATton, P. Sulivan, V.E. OstasheV, 2004: Acoustic tomography array simulation. - Proc. 11th Intern. Symp. on Long Range Sound Propagation, 293-312 (Fairlee, VT).

Cox, E.F., 1949: Abnormal audibility zones in long distance propagation through the atmosphere. - J. Acoust. Soc. Amer. 21, 6-16.

CRARY, A.P., 1950: Stratosphere winds and temperatures from acoustical propagation studies. - J. Meteorol. 7, 233242.

DUKKERT, P., 1931: Über die Ausbreitung von Explosionswellen in der Erdatmosphäre. - Akademische Verlagsgesellschaft, Leipzig.

Gibbons, S., F. Ringdal, T. KVÆrna, 2007: Joint seismic-infrasonic processing of recordings from repeating source of atmospheric explosions. - J. Acoust. Soc. Amer. 122, EL158-164.

Greenfield, R.J., M. Teufel, D.W. Thomson, R. Coulter, 1974: A method for measurement of temperature profiels from refractive transmission of sound. - J. Geophys. Res. 79, 5551-5554.

Holstein, P., A. RaAbe, R. Müller, M. Barth, D. MACKenZIE, E. STARKE, 2004: Acoustic tomography on the basis of travel-time measurements. - Meas. Sci. Technol. 15, 1420-1428.

Johnson, S.A., J.F. GreEnleAf, C.R. HANSEN, W.F. SamayoA, M. TANAKA, A. LEnT, D.A. Christensen, R.L. WOOLLEY, 1977: Reconstructing three dimensional fluid velocity vector fields from acoustic transmission measurements. - In: KESSLER, L.W. (Ed.): Acoustic Holography. Plenum 7, New York. 307-326.

Jovanovic, I., L. Sbaiz, M. VetTerli, 2006: Acoustic tomography method for measuring temperature and wind velocity. - IEEE Intern. Conf. on Acoust., Speech and Signal Processing (Toulouse, France).

KAIMAL, J.C., J.E. CAYNOR, 1983: The Boulder Atmospheric Observatory. - J. Climate Appl. Meteor. 22, 863880.

KulichKov, S.N., G.A. Bush, 2001: Rapid variations in infrasonic signals at long distances from one-type explosions. - Izvestiya, Atm. Ocean. Phys. 37, 306-313.

Links, J.M., J.L. Prince, S.N. GuPTA, 1996: A vector Wiener filter for dual-radionuclide imaging. - IEEE Trans. Med. Imaging 15, 700-709.

MCKENNA, M.H., B.W. STUMP, S. HAYEK, J.R. MCKenNA, T.R. Stanton, 2007: Tele-infrasonic studies of hard-rock mining explosions. - J. Acoust. Soc. Amer. 122, 97-106.

OSTASHEV, V.E., 1982: Returned-inclined acoustic sounding of the atmosphere. - Izv. Acad. Scienc. USSR. Atmos. Ocean. Phys. 18, 695-698.

—, 1997: Acoustics in Moving Inhomogeneous Media. E\&F Spon, London.

Ostashev, V.E., A.G. Voronovich, D.K. Wilson, 2000: Acoustic tomography of the atmosphere. - IEEE Intern. Geoscience and Remote Sensing Symposium, 11861188. 
OstasheV, V.E., S.N. Vecherin, D.K. Wilson, S.L. COLLIER, 2006a: Correlation functions of temperature and velocity fluctuations in a turbulent atmosphere. - J. Acoust. Soc. Amer. 119, 3264.

Ostashev, V.E., S.N. Vecherin, D.K. Wilson, A. ZIEMANN, M. BARTH, K. ARNOLD, 2006b: Acoustic traveltime tomography of temperature and wind velocity fields in the atmosphere. - J. Acoust. Soc. Amer. 120, 3336.

Ostashev, V.E., A. Bedard, S.N. Vecherin, D.K. WILSON, 2008: Acoustic tomography of the atmosphere at the Boulder Atmospheric observatory. - J. Acoust. Soc. Amer. 124, 2591.

OzKan, M.K., A.T. Erdem, M.I. Sezan, A.M. TeKalP, 1992: Efficent multiframe Wiener restoration of blurred and noisy image sequences. - IEEE Trans. Image Process. 1, 453-476.

PIERCE, A.D., J.W. Posey, E.F. ILIFF, 1971: Variation of nuclear explosion generated acoustic-gravity wave forms with burst height and with energy yield. - J. Geophys. Res. 76, 5025-5041.

RAABE, A., S. RAASCh, K. ARnold, M. Letzel, S. Weinbrecht, A. ZiEMANN, 2003: Akustische Tomography der atmosphärischen Grenzschicht zur Validierung eines LES Modells. - Abschlussbericht zu den durch die DFG geförderten Projekten RA 569/4-1,2 und RA 617/41,2 (Leipzig und Hannover).

RaAbe, A., K. Arnold, A. Ziemann, M. Schröter, S. RaAsch, J. Bange, P. ZitTel, Th. Spiess, Th. FoKEN, M. GÖCKEDE, F. BEYRICH, J.-P. LEPS, 2005 STINHO - STructure of turbulent transport under INHOmogeneous conditions - the micro-a scale field experiment and LES modeling. - Meteorol. Z. 14, 315-327.

SPIESBERGER, J.L., 1999: Locating animals from their sounds and tomography of the atmosphere: Experimental demonstration. - J. Acoust. Soc. Amer. 106, 837-846.

SpiesberGer, J.L., K.M. FristruP, 1990: Passive localization of calling animals and sensing of their acoustic environment using acoustic tomography. - Amer. Nat. 135 , 107-153.

Sullivan, P.P., J.C. MCWilliams, C.H. Moeng, 1994: A subgrid-scale model for large-eddy simulation of planetary boundary layer flows. - Bound.-Layer Meteor. 71, 247-276.

TATARSKII, V.I., 1971: The Effects of the Turbulent Atmosphere on Wave Propagation. - Keter, Jerusalem.

Tetzlaff, G., K. Arnold, A. RaAbe, A. Ziemann, 2002: Observations of area averaged nearsurface wind- and temperature fields in real terrain using acoustic travel time tomography. - Meteorol. Z. 11, 273-283.

VECHERIN, S.N., 2007: Acoustic travel-time tomography of the atmosphere. - PhD Thesis, New Mexico State University.

Vecherin, S.N., V.E. Ostashev, D.K. Wilson, A.G. Voronovich, G.H. Goedecke, S.L. Collier, J.M. Noble, D. Ligon, 2004: Forward and inverse problems of acoustic tomography of the atmosphere. - Proc. 11th Intern. Symp. on Long Range Sound Propagation, 293-312 (Fairlee, VT).
VeCHERIN, S.N., V.E. OstasheV, G.H. Goedecke, D.K. Wilson, A.G. Voronovich, 2006a: Timedependent stochastic inversion in acoustic travel-time tomography of the atmosphere. - J. Acoust. Soc. Am. 119, 2579-2588.

Vecherin, S.N., V.E. OstasheV, G.H. Goedecke, D.K. Wilson, A. ZiemanN, M. BARTH, K. ARNOLD, 2006b: Reconstruction of turbulent fields in acoustic tomography experiments. - Proc. 12th Intern. Symp. on Long Range Sound Propagation, 364-384 (New Orleans, LI).

Vecherin, S.N., V.E. Ostashev, A. Ziemann, D.K. Wilson, K. ARnOLD, M. BARTH, 2007: Tomographic reconstruction of atmospheric turbulence with the use of time-dependent stochastic inversion. - J. Acoust. Soc. Am. 122, 1416-1425.

VeCherin, S.N., V.E. OStasheV, D.K. Wilson, 2008a: Three-dimensional acoustic travel-time tomography of the atmosphere. - Acust. Acta Acust. 94, 349-358.

VECHERIN, S.N., V.E. OSTASHEV, D.K. WILSON, A.ZIEMANN, 2008b: Time-dependent stochastic inversion in acoustic tomography of the atmosphere with reciprocal transmission. - Meas. Sci. Technol. 19, 125501, DOI 10.1088/0957-0233/19/12/125501.

WEINBRECHT, S., S. RAASCH, A. ZIEMANN, K. ARNOLD, A. RAABE, 2004: Comparison of large-eddy simulation data with spatially averaged measurements obtained by acoustic tomography - presuppositions and first results. Bound.-Layer Meteor. 111, 441-465.

WILSON, D.K., D.W. ThOMSON, 1994: Acoustic tomographic monitoring of the atmospheric surface layer. - J. Atmos. Ocean. Tech. 11, 751-769.

WILsON, D.K., A. ZIEMANn, V.E. Ostashev, A.G. VORONOVICH, 2001: An overview of acoustic travel-time tomography in the atmosphere and its potential applications. - Acust. Acta Acust. 87, 721-730.

Wilson, D.K., V.E. Ostashev, S.N. Vecherin, A.G. Voronovich, S.L. COllier, J.M. Noble, 2004: Assessment of acoustic travel-time tomography of the atmospheric surface layer. - Proc. AMS Symposium on Boundary Layers and Turbulence (Portland, ME).

Wilson, D.K., E.L. Andreas, J.W. Weatherly, C.L. PetTit, E.G. PATTON, P.P. SUllivan , 2007: Characterization of uncertainty in outdoor sound propagation predictions. - J. Acoust. Soc. Amer. 121, EL177-EL183.

Wilson, D.K., V.E. Ostashev, G.H. Goedecke, 2008: Quasi-wavelet formulations of turbulence and other random fields with correlated properties. - Probabilistic Engineering Mechanics, published online, DOI:10.1016/j.probengmech.2008.09.002.

ZiEmann. A., K. ARnOld, A. RaABE, 1999: Acoustic travel-time tomography - a method for remote sensing of the atmospheric surface layer. - Meteor. Atmos. Phys. 71, 43-51.

,,---2001 : Acoustic tomography as a method to identify smallscale land surface characteristics. - Acust. Acta Acust. 87, 731-737.

,,,--- 2002 : Acoustic tomography as a remote sensing method to investigate the near surface atmospheric boundary layer in comparison with in situ measurememts. - $\underline{\mathbf{J}}$. Atmos. Oceanic Technol. 19, 1208-1215. 